\title{
Manifestações orais provenientes de distúrbios alimentares e a importância da abordagem multidisciplinar
}

Oral manifestations arising from eating disorders and the importance of the multidisciplinary approach

Manifestaciones orales por trastornos de la alimentación y la importancia del enfoque multidisciplinar Faculdade Brasileira Multivix, Brasil E-mail: bubruna06@gmail.com

Bianca Amaral Ferreira de Araújo ORCID: https://orcid.org/0000-0002-0385-6854 Instituto Metropolitano de Ensino Superior, Brasil E-mail: bianca.a@hotmail.com

Amabyli Soares de Souza ORCID: https://orcid.org/0000-0002-1728-0032 Universidade do Extremo Sul Catarinense, Brasil E-mail: amabilysouza1@ gmail.com Ana Auxiliadora de Matos dos Santos ORCID: https://orcid.org/0000-0002-0411-326X Fundação Oswaldo Cruz, Brasil

E-mail: anaauxiliadora3467@gmail.com

Priscilla de Araújo Pereira Monteiro ORCID: https://orcid.org/0000-0002-9021-3661 Fundação Oswaldo Cruz, Brasil

E-mail: enf.priscillamonteirosf@gmail.com

\section{Resumo}

Os distúrbios alimentares são condições de comportamento que interferem de forma negativa na saúde geral e no bem-estar de um indivíduo, sendo a Anorexia Nervosa e a Bulimia Nervosa as desordens mais comuns. Os transtornos alimentares manifestam-se de forma sistêmica devido o comprometimento do estado nutricional e o mesmo ocorre na 
cavidade oral. Diante do exposto, este artigo possui como objetivo revisar a literatura acerca da saúde bucal em pacientes que possuem transtornos alimentares e apontar quais são as manifestações orais mais comuns nesses pacientes. Para a construção deste artigo foi feito um levantamento bibliográfico nas bases de dados Literatura LatinoAmericana e do Caribe em Ciências da Saúde (Lilacs), Scientific Eletronic Library Online (Scielo) e U.S. National Library of Medicine (PUBMED. Foram selecionados artigos contemplados entre os anos de 2010 a 2021.0 levantamento bibliográfico revelou que as manifestações orais mais comuns em pacientes que possuem algum tipo de transtorno alimentares são a erosão dentária, doença cárie, sensibilidade dentinária e xerostomia. O manejo multiprofissional se torna imprescindível para o correto tratamento da causa das lesões orais. Na equipe de saúde deve conter, além dos dentistas, médicos, nutricionistas, psicólogos e, em alguns casos, o psiquiatra, a fim de evitar danos futuros à saúde e garantir bem-estar geral do paciente.

Palavras-chave: Distúrbios alimentares; Bulimia nervosa; Saúde bucal.

\begin{abstract}
Eating disorders are behavioral conditions that negatively interfere with the general health and well-being of an individual, with Anorexia Nervosa and Bulimia Nervosa being the most common disorders. Eating disorders manifest themselves in a systemic way due to compromised nutritional status and the same occurs in the oral cavity. Given the above, this article aims to review the literature on oral health in patients who have eating disorders and point out which are the most common oral manifestations in these patients. For the construction of this article, a bibliographic survey was carried out in the Latin American and Caribbean Literature in Health Sciences (LILACS), Scientific Electronic Library Online (Scielo) and US National Library of Medicine (PUBMED) databases. the years 2010 to 2021. The bibliographic survey revealed that the most common oral manifestations in patients who have some type of eating disorder are dental erosion, caries disease, dentinal sensitivity and xerostomia. cause of oral lesions. The health team must include, in addition to dentists, physicians, nutritionists, psychologists and, in some cases, the psychiatrist, in order to prevent future damage to health and ensure the patient's general well-being.
\end{abstract}

Keywords: Eating disorders; Nervous bulimia; Oral health.

\title{
Resumen
}

Los trastornos alimentarios son afecciones del comportamiento que interfieren negativamente con la salud y el bienestar general de un individuo, siendo la anorexia nerviosa y la bulimia nerviosa los trastornos más comunes. Los trastornos alimentarios se manifiestan de forma sistémica por el estado nutricional comprometido y lo mismo ocurre en la cavidad bucal. Dado lo anterior, este artículo tiene como objetivo revisar la literatura sobre salud bucal en pacientes con trastornos de la conducta alimentaria y señalar cuáles son las manifestaciones bucales más frecuentes en estos pacientes. Para la construcción de este artículo se realizó un relevamiento bibliográfico en las bases de datos de Literatura Latinoamericana y del Caribe en Ciencias de la Salud (LILACS), Scientific Electronic Library Online (Scielo) y Biblioteca Nacional de Medicina de los Estados Unidos (PUBMED). De los años 2010 a 2021. El relevamiento bibliográfico reveló que las manifestaciones bucales más comunes en pacientes que presentan algún tipo de trastorno de la conducta alimentaria son erosión dental, enfermedad de caries, sensibilidad dentinaria y xerostomía. Causa de lesiones bucales. El equipo de salud debe incluir, además de odontólogos, médicos, nutricionistas, psicólogos y, en algunos casos, el psiquiatra, con el fin de prevenir futuros daños a la salud y asegurar el bienestar general del paciente.

Palabras clave: Trastornos de la alimentación; Bulimia nerviosa; Salud bucal.

\section{Introdução}

Os distúrbios alimentares são condições de comportamento que interferem de forma negativa na saúde geral e no bem-estar de um indivíduo (Treasure et al., 2020). A etiologia dessa patologia é distinta, podendo acometer pessoas independente da fase de vida, classe social ou gênero, porém tem sido constatado que o grupo de pessoas formado por adolescente e jovens do sexo feminino são os mais susceptíveis (Jafri et al., 2021).

Os distúrbios alimentares mais comuns são a Anorexia Nervosa e a Bulimia Nervosa. A Anorexia caracteriza-se pela distorção da autoimagem do anoréxico e pelo receio extremo em ganhar peso, resultando na recusa do indivíduo em se alimentar (Melles et al., 2021). Já a Bulimia Nervosa é marcada pela compulsão alimentar seguida por arrependimento da alimentação, fazendo com que o portador dessa patologia tenha comportamentos compensatórios inadequados, a fim de evitar o ganho de peso (Herman \& Bajaka, 2021). Esses comportamentos envolvem o vômito autoinduzido, uso inadequado de laxantes e práticas de exercícios físicos de forma exagerada (Dalle Grave et al., 2021). 
Embora o comportamento dos pacientes portadores desses distúrbios seja diferente, existe semelhança nos sintomas dessas patologias. A forma na qual o indivíduo se enxerga encontra-se distorcida e o desejo pelo corpo perfeito são características marcantes para ambos transtornos (Lima et al., 2012).

Os transtornos alimentares se manifestam de forma sistêmica devido o comprometimento do estado nutricional e o mesmo ocorre na cavidade oral (Casamassimo et al., 2018). Existem algumas características clínicas e sintomatológicas que podem ser observadas na cavidade oral desses pacientes, como erosão dentária, sensibilidade dentinária, doença cárie e comprometimento da glândula parótida (Thomas \& Mirowski, 2010).

Diante do exposto, este artigo possui como objetivo revisar a literatura acerca da saúde bucal em pacientes que possuem transtornos alimentares e apontar quais são as manifestações orais mais comuns nesses pacientes, enfatizando a importância da multidisciplinariedade como forma de garantir a saúde e bem-estar geral desses pacientes.

\section{Metodologia}

Refere-se a uma revisão integrativa de literatura, de caráter qualitativa. A revisão de literatura permite a busca aprofundada dentro de diversos autores e referenciais sobre um tema específico, nesse caso, as manifestações orais provenientes de distúrbios alimentares e a importância da abordagem multidisciplinar (Pereira et al., 2018).

Para a construção deste artigo foi feito um levantamento bibliográfico nas bases de dados Literatura LatinoAmericana e do Caribe em Ciências da Saúde (Lilacs), Scientific Eletronic Library Online (Scielo) e U.S. National Library of Medicine (PUBMED). Os artigos foram coletados no período de março a julho de 2021. Foram selecionados artigos contemplados entre os anos de 2010 a 2021, utilizando os seguintes descritores contidos na lista dos Descritores em Ciência da Saúde (DeCS): "Distúrbios Alimentares", "Bulimia Nervosa", "Saúde Bucal”.

Considerou-se como critério de inclusão os artigos completos disponíveis na íntegra, nos idiomas inglês e português. Os critérios de exclusão foram: artigos incompletos e que não possuem relação com o objetivo do presente estudo.

\section{Resultados e Discussão}

O levantamento bibliográfico revelou que as manifestações orais mais comuns em pacientes que possuem algum tipo de transtorno alimentares são a erosão dentária, doença cárie, sensibilidade dentinária e xerostomia (Thomas \& Mirowski, 2010).

\subsection{Erosão dentária}

A erosão dentária consiste na desmineralização patológica da matriz inorgânica da estrutura dentária causada por processo químico não bacteriano (Catelan et al., 2010). É a manifestação oral mais comum em pacientes que possuem o hábito do vômito autoinduzido, provocado pelo distúrbio alimentar como bulimia, anorexia e, ainda, em pacientes com refluxo (Li et al., 2017).

Nesses casos, há o retorno do ácido gástrico para a cavidade oral. A gravidade da erosão dentária depende da quantidade de vezes que o paciente provoca o vômito, hábitos de higiene oral e se o paciente faz enxague bucal com agua ou líquidos neutralizantes, como o leite. Juntamente, os ácidos de origem externa, provenientes de alimentos ácidos, bebidas ou medicamentos podem interferir no grau da erosão dentária (Kannan et al., 2014).

A saliva possui capacidade tampão e, portanto, as superfícies mais afetadas são aquelas que não estão protegidas pela secreção serosa, sendo elas: faces vestibulares e palatinas dos dentes localizados na arcada superior e as superfícies vestibulares e oclusais dos dentes posteriores da arcada inferior (Faine, 2003). A erosão dentária pode ser notada 6 meses após 
o início do vômito (Marshall, 2018). Sendo assim, o profissional de saúde, mais especificamente o dentista, deve estar apto a reconhecer as manifestações clínicas deste tipo de desordem e conduzir o melhor tratamento para o paciente.

\subsection{Cárie dentária}

A cárie dentária é uma das doenças mais prevalentes em todo o mundo e consiste na desmineralização da superfície do dente, provocado pelo ataque ácido de bactérias (Dayo et al., 2021). Possui etiologia multifatorial, porem está intimamente associada aos hábitos de vida do indivíduo. Pacientes que possuem dieta irregular, principalmente com consumo excessivo de açúcar são mais propensos a desenvolverem a cárie dentária (Alshammari et al., 2021).

Há conflitos na literatura acerca da relação entre cárie dentária e os transtornos alimentares, alguns autores afirmam que a incidência de cárie em indivíduos com essas patologias não se encontra destoantes daquela encontrada em pacientes considerados saudáveis. Em contrapartida, alguns pesquisadores relatam que há aumento nas taxas de cárie, devido ao fato de pacientes com bulimia possuírem uma dieta rica em açúcar e carboidratos (Thomas \& Mirowski, 2010).

\subsection{Sensibilidade dentinária}

A sensibilidade dentinária ocorre quando há exposição dos túbulos dentinários, causados pelo desgaste excessivo do esmalte do dente, ocasionado pela doença cárie, abrasão, erosão e abfrasão, tornando a superfície dentária sensível a estímulos táteis, térmicos ou físicos, sendo fumantes e pacientes com bulimia o grupo de risco para esse tipo de acometimento (Magloire, 2011).

\subsection{Xerostomia}

A xerostomia consiste de uma sensação subjetiva de boca seca, podendo estar associada, ou não, à hiposalivação. Os pacientes que possuem transtornos alimentares geralmente se utilizam de terapia medicamentosa, como ansiolíticos e antidepressivos, que provocam alguns afeitos colaterais manifestados na cavidade oral, como a xerostomia (Silva et al., 2021).

A saliva possui papel tampão e contribui com a limpeza da cavidade bucal pela sua atividade antimicrobiana e quando há alteração no fluxo salivar, os processos fisiológicos encontram-se prejudicados, colaborando para o desenvolvimento da cárie dentária, além de causar desconfortos como dor, dificuldade na mastigação e deglutição (Djordjevic et al., 2016).

\subsection{A importância da multidisciplinaridade}

Além das alterações sistêmicas, os distúrbios alimentares trazem prejuízos na cavidade oral. Em alguns casos, a primeira manifestação de distúrbios alimentares ocorre na boca, então o cirurgião dentista deve estar atento aos sinais e sintomas, além de exercer uma anamnese rica em detalhes, a fim de identificar o agente etiológicos das desordens bucais citadas e evitar danos futuros a saúde e garantir bem-estar geral do paciente (Ruiz-Ramos et al., 2021).

O profissional deve estar apto a diagnosticar as lesões já existentes, prevenir lesões futuras e contribuir para a remoção da causa dos distúrbios alimentares, encaminhando o paciente para outros profissionais, quando se fizer necessário (Thomas \& Mirowski, 2010).

O manejo multiprofissional se torna imprescindível para o correto tratamento da causa das lesões orais. Na equipe de saúde deve conter, além dos dentistas, médicos, nutricionistas, psicólogos e, em alguns casos, o psiquiatra (Gance-Cleveland \& Ozkaynak, 2021). 


\section{Conclusão}

O presente estudo objetivou, por meio de uma revisão bibliográfica, avaliar a saúde oral em pacientes que possuem transtornos alimentares e apontar quais são as manifestações orais mais comuns nesses pacientes.

O levantamento bibliográfico constatou que a erosão dentária é a manifestação clínica mais encontrada em pacientes que possuem distúrbios alimentares, porém a cárie dentária, sensibilidade dentinária e xerostomia também possuem associação com esses transtornos. O manejo multiprofissional se torna imprescindível para o correto tratamento da causa das lesões orais. Na equipe de saúde deve conter, além dos dentistas, médicos, nutricionistas, psicólogos e, em alguns casos, o psiquiatra.

Com isso, a revisão de literatura sobre as manifestações orais provenientes de distúrbios alimentares e a importância da abordagem multidisciplinar se mostra importante, pois engloba os artigos anteriores que possuem relação com o tema proposto e, ao mesmo tempo, cria-se possibilidade futura de novos pesquisadores abordarem o tema, criando novos trabalhos relevantes, para que haja melhora na qualidade de vida de pacientes com transtornos alimentares.

\section{Referências}

Alshammari, F. R., Alamri, H., Aljohani, M., Sabbah, W., O’Malley, L., \& Glenny, A.-M. (2021). Dental caries in KSA: A systematic review. Journal of Taibah University Medical Sciences. https://doi.org/https://doi.org/10.1016/j.jtumed.2021.06.008

Casamassimo, P. S., Flaitz, C. M., Hammersmith, K., Sangvai, S., \& Kumar, A. (2018). Recognizing the Relationship Between Disorders in the Oral Cavity and Systemic Disease. Pediatric Clinics of North America, 65(5), 1007-1032. https://doi.org/https://doi.org/10.1016/j.pcl.2018.05.009

Catelan, A., Paula, A., \& Guedes, A. (2010). Erosão dental e suas implicações sobre a saúde bucal Dental erosion and its implications on the oral health. 8386.

Dalle Grave, R., Sartirana, M., Sermattei, S., \& Calugi, S. (2021). Treatment of Eating Disorders in Adults Versus Adolescents: Similarities and Differences. Clinical Therapeutics, 43(1), 70-84. https://doi.org/https://doi.org/10.1016/j.clinthera.2020.10.015

Dayo, A. F., Wolff, M. S., Syed, A. Z., \& Mupparapu, M. (2021). Radiology of Dental Caries. Dental Clinics of North America, 65 (3), $427-445$. https://doi.org/https://doi.org/10.1016/j.cden.2021.02.002

Djordjevic, V., Djokic, G., Domic, D., Zivkovic, N., Jankovic, L., Milicic, B., \& Dejanovic, S. D. (2016). P.3.d.013 - Hyposalivation and xerostomia in schizophrenic patients on psychotropic medications. European Neuropsychopharmacology, 26, S537. https://doi.org/https://doi.org/10.1016/S0924977X(16)31576-0

Faine, M. P. (2003). Recognition and management of eating disorders in the dental office. Dental Clinics of North America, 47(2), 395-410. https://doi.org/https://doi.org/10.1016/S0011-8532(02)00108-8

Gance-Cleveland, B., \& Ozkaynak, M. (2021). Multidisciplinary Teams are Essential for Developing Clinical Decision Support to Improve Pediatric Health Outcomes: An Exemplar. Journal of Pediatric Nursing, 58, 104-106. https://doi.org/https://doi.org/10.1016/j.pedn.2020.08.012

Herman, A., \& Bajaka, A. (2021). The role of the intestinal microbiota in eating disorders - bulimia nervosa and binge eating disorder. Psychiatry Research, 300, 113923. https://doi.org/https://doi.org/10.1016/j.psychres.2021.113923

Jafri, S., Frykas, T. L., Bingemann, T., Phipatanakul, W., Bartnikas, L. M., \& Protudjer, J. L. P. (2021). Food Allergy, Eating Disorders and Body Image. Journal of Affective Disorders Reports, 6, 100197. https://doi.org/https://doi.org/10.1016/j.jadr.2021.100197

Kannan, A., Adil Ahmed, M. A., Duraisamy, P., Manipal, S., \& Adusumillil, P. (2014). Dental hard tissue erosion rates and soft drinks - A gender based analysis in Chennai city, India. The Saudi Journal for Dental Research, 5(1), 21-27. https://doi.org/https://doi.org/10.1016/j.ksujds.2013.08.003

Li, W., Liu, J., Chen, S., Wang, Y., \& Zhang, Z. (2017). Prevalence of dental erosion among people with gastroesophageal reflux disease in China. The Journal of Prosthetic Dentistry, 117(1), 48-54. https://doi.org/https://doi.org/10.1016/j.prosdent.2016.04.029

Lima, D. S. M. de, Coutinho, V. M., \& Holanda, L. C. A. de. (2012). A saúde oral e os transtornos alimentares entre adolescentes. Revista Brasileira de Odontologia, 69(2), 190-193.

Magloire, H. (2011). Odontoblast and dentin thermal sensitivity. PAIN®, 152(10), 2191-2192. https://doi.org/https://doi.org/10.1016/j.pain.2011.02.042

Marshall, T. A. (2018). Dietary assessment and counseling for dental erosion. The Journal of the American Dental Association, 149(2), 148-152. https://doi.org/https://doi.org/10.1016/j.adaj.2017.11.006

Melles, H., Spix, M., \& Jansen, A. (2021). Avoidance in Anorexia Nervosa: Towards a research agenda. Physiology \& Behavior, $238,113478$. https://doi.org/https://doi.org/10.1016/j.physbeh.2021.113478

Pereira, A., Shitsuka, D., Parreira, F., \& Shitsuka, R. (2018). Método Qualitativo, Quantitativo ou Quali-Quanti. In Metodologia da Pesquisa Científica. https://repositorio.ufsm.br/bitstream/handle/1/15824/Lic_Computacao_Metodologia-Pesquisa-Cientifica.pdf?sequence=1. 
Research, Society and Development, v. 10, n. 10, e357101018965, 2021

(CC BY 4.0) | ISSN 2525-3409 | DOI: http://dx.doi.org/10.33448/rsd-v10i10.18965

Ruiz-Ramos, J., Hernández, M. H., Juanes-Borrego, A. M., Milà, R., Mangues-Bafalluy, M. A., \& Mestres, C. (2021). The Impact of Pharmaceutical Care in Multidisciplinary Teams on Health Outcomes: Systematic Review and Meta-Analysis. Journal of the American Medical Directors Association. https://doi.org/https://doi.org/10.1016/j.jamda.2021.05.038

Silva, I. M. V, Donaduzzi, L. C., Perini, C. C., Couto, S. A. B., Werneck, R. I., de Araújo, M. R., Kurahashi, M., Johann, A. C. B. R., Azevedo-Alanis, L. R., Vieira, A. R., \& Couto-Souza, P. H. (2021). Association of xerostomia and taste alterations of patients receiving antineoplastic chemotherapy: A cause for nutritional concern. Clinical Nutrition ESPEN, 43, 532-535. https://doi.org/https://doi.org/10.1016/j.clnesp.2021.03.006

Thomas, D. M., \& Mirowski, G. W. (2010). Nutrition and oral mucosal diseases. Clinics in Dermatology, 28(4), 426-431. https://doi.org/https://doi.org/10.1016/j.clindermatol.2010.03.025

Treasure, J., Duarte, T. A., \& Schmidt, U. (2020). Eating disorders. The Lancet, 395(10227), 899-911. https://doi.org/https://doi.org/10.1016/S01406736(20)30059-3 